\title{
Drug Utilization Pattern of Antibiotics: The Role of Age, Sex and Municipalities in Determining Variation
}

This article was published in the following Dove Press journal: Risk Management and Healthcare Policy

\author{
Valentina Orlando $\mathbb{D}^{\prime}$ \\ Valeria Marina Monetti (iD) \\ Aida Moreno Juste (iD ${ }^{2,3}$ \\ Veronica Russo (D) ${ }^{1}$ \\ Sara Mucherino' \\ Ugo Trama ${ }^{4}$ \\ Antonella Guida (iD) \\ Enrica Menditto (D)
}

'CIRFF, Center of Pharmacoeconomics, University of Naples Federico II, Naples, Italy; ${ }^{2}$ Aragon Health Sciences Institute (IACS), IIS Aragón, REDISSEC ISCIII, Zaragoza, Spain; ${ }^{3}$ Aragon Health Service (SALUD), Zaragoza, Spain; ${ }^{4}$ Regional Pharmaceutical Unit, Campania Region, Naples, Italy; ${ }^{5}$ Directorate-General for Protection of Health, Campania Region, Naples, Italy
Correspondence: Valentina Orlando CIRFF, Center of Pharmacoeconomics, University of Naples Federico II, Via Domenico Montesano 49, Naples 80I3I, Italy

Tel +39081678657

Fax +39081678303

Email valentina.orlando@unina.it
Purpose: The purpose was to analyze drug prescription and antibiotic use by age and sex in Italy's Campania Region, and to estimate the distribution of prescription rates in children $(\leq 14$ years old), adults (between 15 and 65 years old), and older adults ( $\geq 65$ years old) at a municipality level.

Methods: This was a retrospective analysis of pharmacy records in Campania (Southern Italy), in 2016. Difference in antibiotic prescriptions in different age groups was assessed by prevalence rates. Age-adjusted prevalence rates were categorized into quintiles and mapped by the patient's municipality of residence. Relationship between prevalence rates for the different age groups was estimated using the non-parametric Spearman rank correlation test.

Results: There were 2,738,118 were patients with at least one antibiotic prescription. Antibiotics prescription was higher in children aged $<5$ years and in the older adults aged $>70$ years. Prevalence rate distribution was different among municipalities in all age groups. A positive correlation between the rank distribution of prevalence rates at municipality level was identified for children and adults ( $\mathrm{rs}=0.56 ; P<0.01)$, adults and the older adults $(\mathrm{rs}=0.79 ; P<0.01)$, and children and the older adults ( $\mathrm{rs}=0.46 ; P<0.01)$. Among the studied age groups, the most prescribed antibiotic class was penicillin (except the older adults aged $\geq 85$ years) ranging from $45 \%$ in children to $27.2 \%$ in the older adults. Fluoroquinolones were the least prescribed antibiotic class, ranging from $0.2 \%$ in children to $30.2 \%$ in the older adults.

Conclusion: A considerably high use of antibiotic drugs has been detected in Campania Region, with values exceeding the regional and national average. Prescriptions at municipal level differ from one age group to another. Antibiotic use is often unjustified, and to decrease the number of prescriptions and improve their appropriateness, several measures at territorial level are recommended.

Keywords: antibiotic use, drug utilization, prescription patterns, real-world data

\section{Introduction}

Antibiotics are the most prescribed drugs in outpatient populations, ${ }^{1,2}$ but more than $30 \%$ of patients take them unnecessarily. ${ }^{3,4}$ The World Health Organization (WHO) advocates the correct use of antibiotics to avoid antibiotic resistance, which has reached alarming levels across the globe. ${ }^{4}$

Antibiotics are widely prescribed therapeutic agents for children and the older adults in community settings. Although some conditions do not typically benefit from antibiotic therapy, these drugs are frequently used to treat colds and bronchitis 
(which are the most common conditions in children); and bacterial infections such as urinary tract infections (UTIs), pneumonia, and skin/soft tissue infections (common in the older adults). ${ }^{5}$ It has been estimated that nearly $50 \%$ of children's antibiotic prescriptions are unnecessary ${ }^{6}$ and do not comply with national guidelines. ${ }^{7}$

Antibiotic misuse causes unnecessary expenditure, overuse of health services (as patients keep consulting their general practitioners [GPs] for subsequent similar problems), unnecessary side effects, and the possible development of antibiotic resistance. ${ }^{6,7}$ Thus, antibiotic misuse and overuse impact many aspects of public health. ${ }^{6}$

Different antibiotic prescribing patterns exist according to age and geographical settings. ${ }^{5}$ Indeed, differences in antibiotic prescription rates were found not only between different countries but also at a regional level. In Europe, these geographical variations have been attributed to socioeconomic (eg, financial wellbeing and access to health insurance), sociodemographic (eg, urbanization), and cultural (eg, educational level, prescribing norms, and patient demands) factors. ${ }^{8,9}$ Furthermore, there is evidence that antibiotic prescription rates vary considerably according to age and sex. ${ }^{5,10}$ In Italy, where the consumption of systemic antibiotics is higher than the European average, $2,11,12$ antibiotic prescription rates vary among different regions, 5,13,14 showing a higher antibiotic consumption in Southern Italy. Particularly, Campania is the region with the highest antibiotic consumption. $^{15}$ According to earlier estimates of a recent study, in this region, there is a strong relationship between antibiotic prevalence rates and sociodemographic and socioeconomic factors at a municipality level. ${ }^{16}$ Nevertheless, this work did not analyze prescriptive patterns related to age, sex and type of class of antibiotic drugs. Reason why, the aims of the present study are to (a) analyze outpatient drug prescriptions records in Campania to describe patterns of antibiotic use by age and sex, and (b) estimate the distribution of prevalence prescription rates in children, adults, and the older adults $\geq 65$ years at a municipality level.

\section{Materials and Methods}

\section{Setting}

The Italian National Health Service (NHS) has been decentralized at national, regional, and local level since 2001. ${ }^{17}$ Campania, one of the largest regions of southern Italy, had a population of 5,850,850 inhabitants up to 1 January $2016 .^{18}$ As all other Italian regions, it provides health care services (free or at a nominal charge) to all citizens and legal foreign residents through Local Health Units (LHUs). Each LHU corresponds to a geographic area in Campania, which are five: Naples (including three LHUs: Na1, Na2, Na3), Avellino (Av-LHU), Benevento (Bn-LHU), Caserta (CeLHU), and Salerno (Sa-LHU). Each one is constituted by healthcare districts, which aggregate different municipalities. Overall there are 550 municipalities.

\section{Data Source}

For the present study, we analysed pharmacy claims databases of Campania region. These databases contain records of all drugs dispensed by retail pharmacies and reimbursed by the NHS, information regarding the patient's identification code, drug code, number of Defined Daily Doses (DDD), formulation, number of packages for each claim, date of prescription, date of dispensation, and drug price. All drugs were classified according to the Anatomical Therapeutic Chemical (ATC) classification system. ${ }^{19}$

Pharmacy claims databases were linked to civil registries, containing demographic information (ie, age, sex, LHU and municipality of residence) of all residents covered by the Regional Health System (RHS). The above databases had been previously validated and used to produce drug-utilization information. ${ }^{20-25}$

Data sources were matched by record linkage analysis through a unique and anonymous personal identification code. Such code was created by a database manager uninvolved in the data analysis, preventing patient identification. Therefore, informed consent forms were not required.

\section{Cohort Definition}

We conducted a descriptive cross-sectional drug use study which included the entire Campania population of $5,850,850$ inhabitants. Noteworthy is that the study sample represented about $10 \%$ of the total Italian population.

The whole studied cohort was divided into three groups (children aged $\leq 14$ years, adults aged between 15 and 65 years, and the older adults aged $\geq 65$ years) and distributed into the 550 municipalities. The entire Campania's population was also stratified by age $(0-6,7-14,15-24,25-34$, $35-44,45-54,55-64,65-74,75-84$, and $\geq 85$ years) and by sex. In our analysis, official data on resident population in the Italian municipalities, which are available on Demo Istat website, were up to date until January $1,2016 .^{18}$

\section{Outcomes}

Prevalence rate was used as a measure to estimate the degree of exposure to antibiotic prescription. 
Prescription data for the year 2016 with all dispensed ATC-code 'J01' drugs were analyzed. Study drugs are listed in Supplementary material. The outpatient parenteral therapy (OPAT) was not included in the analyses.

Antibiotic prevalence rates for the overall treated patients were calculated as the proportion of the population who received at least one prescription of ' $\mathrm{J} 01$ ' drugs (ATC-II level) in 2016. While, antibiotic prevalence rates for patients treated with different drug class (ATC-III level) were calculated as the proportion of the population who received at least one prescription of penicillins, cephalosporins, macrolides, fluoroquinolones and the other drug classes involved in the study, in order to focus on drugs with prevalence rate $>1 \%$.

Prevalence rates were estimated by age and sex.

At municipality level, prevalence rates for adults and the older adults were adjusted by age using a direct standardization method, where the standard population (also known as reference population) was the population in Campania up to January 1, 2016.

\section{Statistical Analysis}

The age-adjusted prevalence rates were categorized into quintiles and mapped by the patient's municipality of residence. Values were presented as mean \pm standard deviation (STD). The coefficient of variation (CV) was also calculated as a measure of dispersion ( $\mathrm{CV}=\mathrm{STD} /$ mean). Confidence intervals (CIs) were not calculated as they were not relevant due to the high number of individuals in the study population. The relationship between prevalence rates for children, adults, and the older adults was estimated using the nonparametric Spearman rank correlation test.

A logistic regression analysis was performed for each of the most common antibiotic classes to evaluate the association between receiving an antibiotic prescription and gender, age group and municipality type.

All analyses were performed using SPSS software version 17.1 for Windows (SPSS Inc, Chicago, IL, USA), and a $p$-value of $<0.05$ was considered to be statistically significant. Maps for antibiotic prevalence rates were generated by a custom script that uses an Application Programming Interface (API) offered by MapBox (www.mapbox.com).

\section{Results}

In 2016, 2,738,118 patients in Campania received at least one antibiotic prescription. The total antibiotic prevalence rate was $46.8 \%, 50.3 \%$ for females and $43.2 \%$ for males. While, the prevalence rates among different age groups, were $43.8 \%$ for children, $42.4 \%$ for adults, and $65.8 \%$ for older adults.
Penicillins were the most commonly prescribed antibiotic class (ie, $57.8 \%$ of the population treated with antibiotics) showing that $27.0 \%$ of the total population in Campania received at least one prescription of this type of drug.

The prescription rate for cephalosporins was $14.3 \%$ of the total population, $13.1 \%$ for macrolides and $12.6 \%$ for fluoroquinolones.

Figure 1 shows Campania's antibiotic prevalence rates regarding age and sex, in 2016.

Antibiotics prescription was higher in children aged $<5$ years and in the older adults aged $>70$ years. Particularly, the highest prevalence rates values were noted for 4-year-old children (60.7\%) and 71-year-old individuals (80.3\%). After the age of 4 , prevalence rates decreased rapidly, reaching the lowest value at 14 years $(31.6 \%)$. Thereafter, rates progressively increased, reaching the highest value at 71 years of age.

Among children, prevalence rates were slightly higher in males than females ( $46.8 \%$ vs $45.4 \%$, respectively). Among adults, females had average antibiotic prevalence rates higher than males $(52.0 \%$ vs $44.7 \%$, respectively). The prevalence rates trend was again inverted in the older adults group ( $70.2 \%$ for males vs $67.5 \%$ for females). The highest prevalence rates value was reached by 71 -year-old females $(81.8 \%)$, while the lowest one was reached by $33-$ year-old males $(29.3 \%)$.

Table 1 shows antibiotic use of the most commonly prescribed antibiotic classes by gender and age group in 2016, as a percentage of people receiving at least one antibiotic prescription.

Penicillins were the most prescribed antibiotic class in all age groups, except for the older adults aged $\geq 85$ years. Regarding the treated population, prevalence rates ranged from $67.9 \%$ in children aged $\leq 6$ years to $45.2 \%$ in the older adults aged $\geq 85$ years. For the class of Fluoroquinolones, the results were clearly different, in fact, they are the class of antibiotics prescribed less frequently in children $\leq 6$ years of age $(0.3 \%)$ while in terms of prescription records higher values in the older adults $(50.0 \%)$. The percentage of patients treated with macrolides gradually decreased from $41.7 \%$ in children aged $\leq 6$ years to $22.0 \%$ in the older adults aged $\geq 85$ years. Finally, the percentage of treated with cephalosporins was similar between children group and the older adults' group, reaching the highest value in the older adults aged $\geq 85$ years $(45.3 \%)$ (Supplementary materials). 


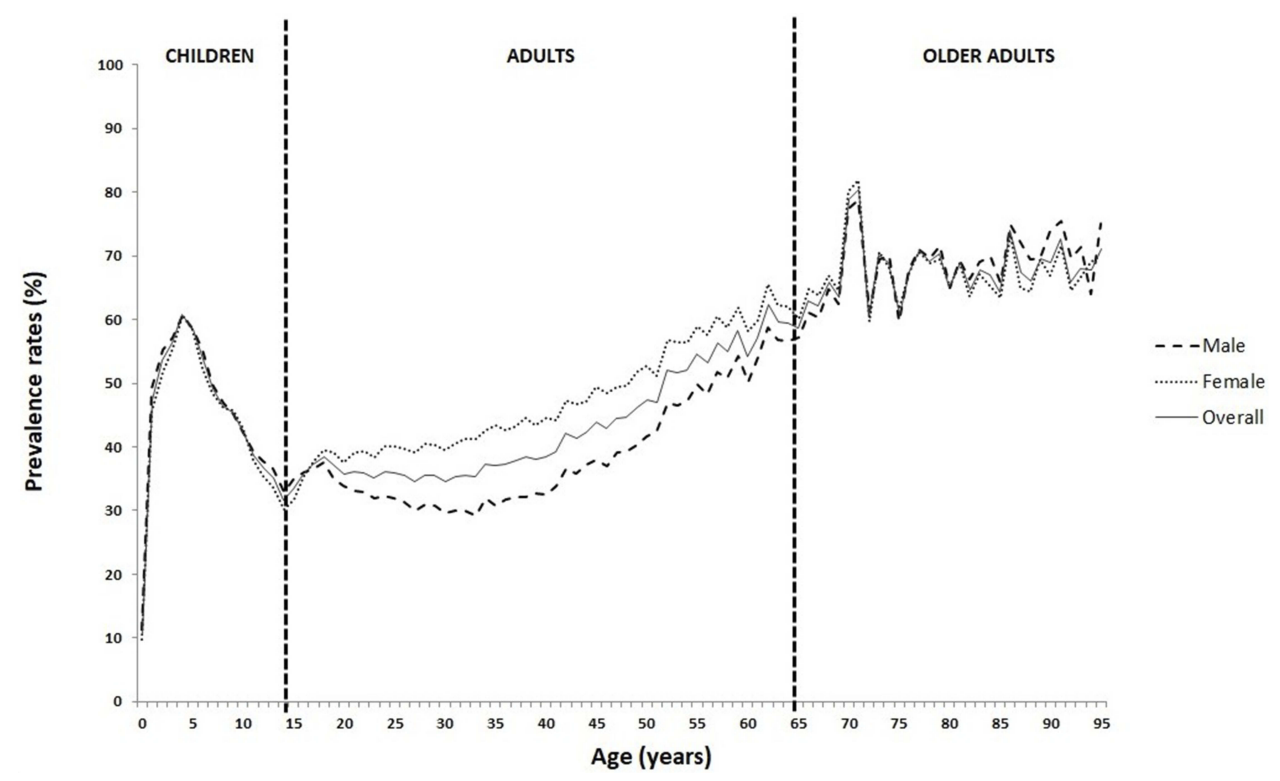

Figure I Antibiotic prevalence rates by age and sex in Campania, 2016.

Patterns of antibiotic use were assessed by using prescrip- $\quad$ from $33.1 \%$ in Benevento to $47.9 \%$ in Naples2 (mean 42.7 tion prevalence rates according to age within each LHU. In $\quad \pm 5.0 \%, \mathrm{CV}=0.12$ ). In adults, they ranged from $37.9 \%$ in children, standardized prevalence rates at LHU level ranged Benevento to $46.1 \%$ in Naples2 (mean $41.9 \pm 2.6 \%$,

Table I Prevalence Rates of Antibiotic Prescription Stratified by Drug Classes and Patients' Characteristics

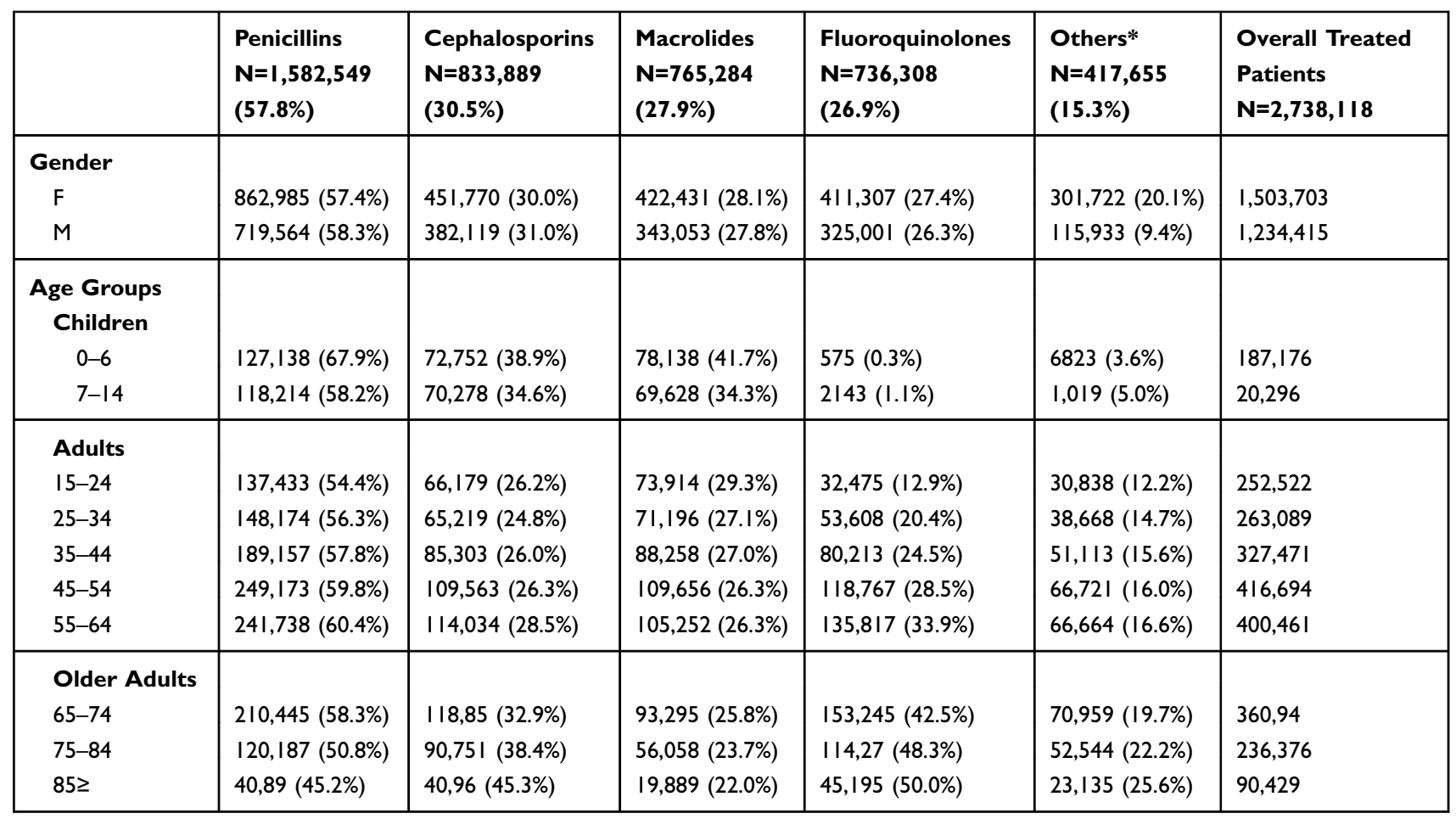

Notes: Drug classes were not mutually exclusive. Prevalence rates were calculated by dividing the number of patients receiving almost one prescription of a specific drug class by the total of patients treated with antibiotics. *Others: JOIAA Tetracyclines, JOIBA Amphenicols, J0IEE combinations of Sulfonamides and Trimethoprim, Incl. derivatives, J0IFF Lincosamides, J0I GB other Aminoglycosides, JOIMB other Quinolones, J0 IXA Glycopeptide Antibacterials, J0 IXD Imidazole derivatives, J0 IXE Nitrofuran derivatives, $J 0 I X X$ other antibacterials. 
$\mathrm{CV}=0.06$ ); and in the older adults, from $59.9 \%$ in Benevento to $71.8 \%$ in Naples2 (mean $65.3 \pm 4.2 \%, \mathrm{CV}=0.06$ ).

Figure 2 shows the distribution of antibiotic prevalence rates, in quintiles by municipality within each LHU, for children (Figure 2A), adults (Figure 2B), and the older adults (Figure 2C). Distribution of prevalence rates differed among the age groups.

Among children, higher rates were observed in the coastal areas around Naples, northern Caserta, and eastern Avellino (50.2-68.0\%). For the Coastal areas around
Naples up to southern Caserta and western Avellino, high prevalence rates were recorded both for adults (46.3-$59.5 \%)$ and the older adults (71.0-89.5\%). Benevento and Salerno showed lower prevalence rates compared to other areas for the three age groups.

For children, prevalence rates reached the lowest value in Corleto Monteforte (Sa-LHU) with $11.3 \%$, and the highest in Guardia Lombardi (Av-LHU) with 68.0\% (mean $41.8 \pm 10.0 \%$ ). For adults, the lowest value was in Omignano (Sa-LHU) with $11.4 \%$ and the highest in

\section{A Children}

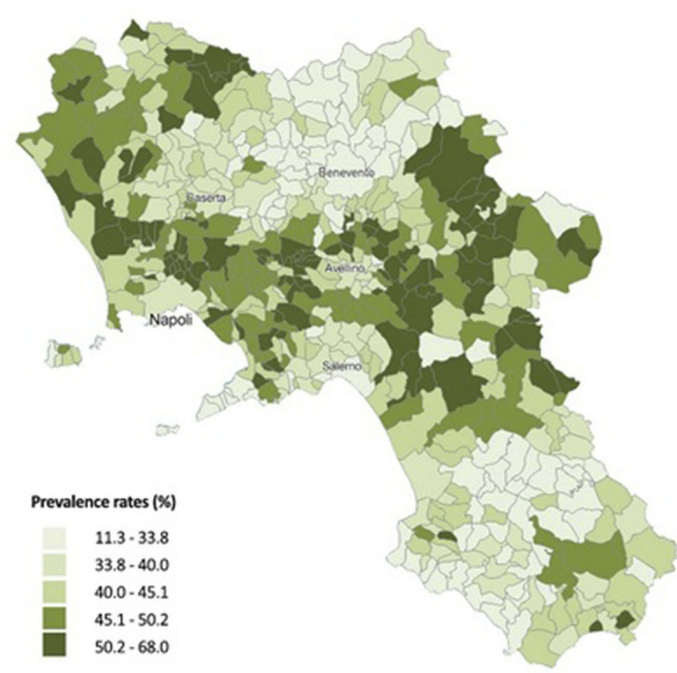

B

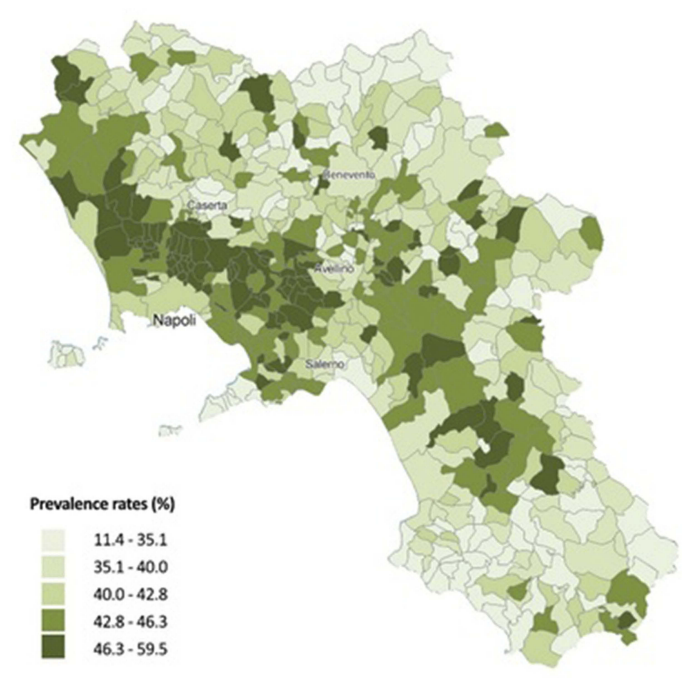

C

Older Adults

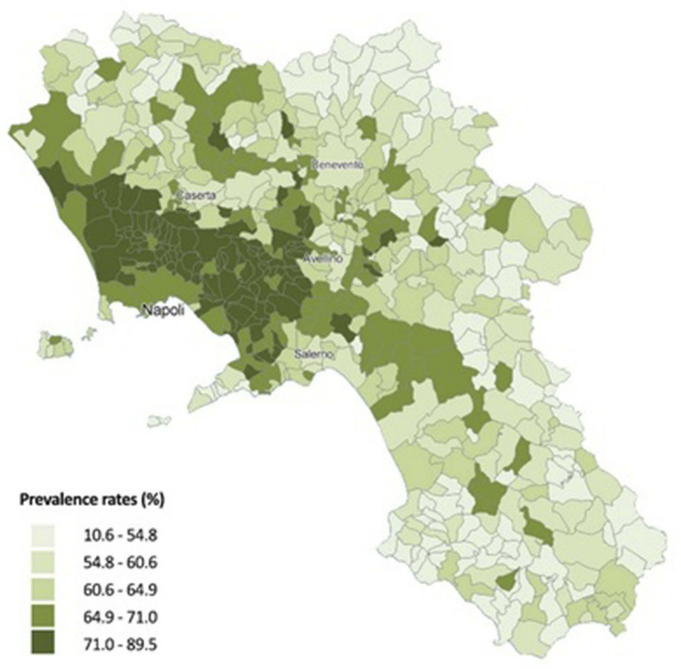

Figure 2 (A) Antibiotic prevalence rates (\%) in children at a municipality level in Campania. (B) Antibiotic prevalence rates (\%) in adults at a municipality level in Campania. (C) Antibiotic prevalence rates (\%) in older adults $\geq 65$ years at a municipality level in Campania. 
Moschiano (Av-LHU) with 59.5\% (mean 40.9 $\pm 6.7 \%$, $\mathrm{CV}=0.16$ ). For the older adults' group, prevalence rates reached the lowest value in Romagnano al Monte (SaLHU) with $10.6 \%$ and the highest in Marzano di Nola (AvLHU) with $89.5 \%$ (mean $62.2 \pm 9.9 \%, \mathrm{CV}=0.16$ ).

We found a statistically significant correlation of the rank distribution (rs) at municipality level of the prevalence rates between children and adults ( $\mathrm{rs}=0.56 ; P<0.01$ ). Furthermore, a positive correlation was also found between adults and the older adults ( $\mathrm{rs}=0.79 ; P<0.01)$, and children and the older adults ( $\mathrm{rs}=0.46 ; P<0.01)$.

Table 2 reports the results of the multivariate analysis for each of the most commonly prescribed antibiotic classes. The age group and municipality type were the main determinants of receiving at least one drug prescription for each of the antibiotic classes. In particular, being a child compared to an adult was the factor associated with the highest risk of drug exposure for penicillins, fluoroquinolones and macrolides (Penicillins, $\mathrm{OR}=1.22$, 95\% C.I. 1.21, 1.23; Macrolides, $\mathrm{OR}=1.66$, 95\% C.I. 1.21, 1.23). Instead, patients older than 65 years old (the older adults age group) compared to adults had a higher risk of receiving at least a prescription of cephalosporins $(\mathrm{OR}=2.48$, $95 \%$ C.I. 2.46, 2.49) and Fluoroquinolones $(\mathrm{OR}=1.57$, 95\% C.I. 1.56, 1.58).

Furthermore, living in an urban municipality also slightly increased the probability of receiving a prescription of penicillins $(\mathrm{OR}=1.09,95 \%$ C.I. 1.08, 1.10), cephalosporins $(\mathrm{OR}=1.08,95 \%$ C.I. 1.07, 1.09) and fluoroquinolones $(\mathrm{OR}=1.08,95 \%$ C.I. $1.08,1.09)$.

\section{Discussion}

In Italy, differences in interregional antibiotic prescribing rates have already been described in the literature, with a higher consumption in southern Italy compared to the northern regions. ${ }^{5,6,10,26}$ However, to the best of our knowledge, this is the first study comparing intraregional differences in antibiotic prescription rates for children, adults, and the older adults at municipality level in Italy. ${ }^{10}$ Particularly, antibiotic consumption in Campania is described as the highest in Italy. ${ }^{15}$

In our study, we found considerable differences at municipality level for antibiotic prevalence rates in children (11.3-68.0\%), adults (11.4-59.5\%), and the older adults (10.6-89.5\%). These data confirmed the results from a previous study conducted in the same cohort of patients, which showed higher prevalence rates in coastal areas around Naples up to southern Caserta and eastern Avellino. Furthermore, the study concluded that higher prevalence rates were related to urban municipalities and low average annual income levels. ${ }^{16}$ In our study, we observed a statistically significant correlation of rank distributions of prevalence rates at municipality level between children, adults, and the older adults. Different factors may explain these geographical differences observed in all three groups, such as socio-cultural, demographic, economic determinants, as well as different prescribing attitudes between physicians. ${ }^{5}$

In 2016, antibiotics represented $8.4 \%$ of the total pharmaceutical expenditure in Campania, with a per capita value of $15 €$. A large variability existed among prevalence prescription rates and the different LHUs, with a lowest expenditure value in Benevento (12€ per capita) and the highest in Naples3 (18€ per capita). ${ }^{15}$

Table 2 Multivariate Regression Analysis of Antibiotic Prescription

\begin{tabular}{|c|c|c|c|c|c|c|c|c|}
\hline \multirow[t]{2}{*}{ Characteristics } & \multicolumn{2}{|l|}{ Penicillins } & \multicolumn{2}{|l|}{ Cephalosporins } & \multicolumn{2}{|l|}{ Fluoroquinolones } & \multicolumn{2}{|l|}{ Macrolides } \\
\hline & $\begin{array}{l}\text { Adjusted OR } \\
(95 \% \mathrm{CI})\end{array}$ & $p$-value & $\begin{array}{l}\text { Adjusted OR } \\
(95 \% \mathrm{CI})\end{array}$ & $p$-value & $\begin{array}{l}\text { Adjusted OR } \\
(95 \% \mathrm{CI})\end{array}$ & $p$-value & $\begin{array}{l}\text { Adjusted OR } \\
(95 \% \mathrm{CI})\end{array}$ & $p$-value \\
\hline \multicolumn{9}{|l|}{ Gender } \\
\hline Female & Reference & & Reference & & Reference & & Reference & \\
\hline Male & $1.039(1.034-1.044)$ & $<0.00 I^{*}$ & $1.034(1.029-1.040)$ & $<0.00 I^{*}$ & $1.024(1.018-1.029)$ & $<0.001 *$ & $0.961(0.956-0.966)$ & $<0.001 *$ \\
\hline \multicolumn{9}{|l|}{ Age Groups } \\
\hline Children & $1.220(1.211-1.229)$ & $<0.00 I^{*}$ & $0.02 \mathrm{I}(0.020-0.02 \mathrm{I})$ & $<0.00 I^{*}$ & $1.531(1.520-1.543)$ & $<0.00 I^{*}$ & $1.655(1.643-1.667)$ & $<0.00 I^{*}$ \\
\hline Adults & Reference & & Reference & & Reference & & Reference & \\
\hline Older adults & $0.826(0.82 I-0.83 I)$ & $<0.001 *$ & $2.477(2.463-2.492)$ & $<0.001 *$ & $1.569(1.560-1.579)$ & $<0.00 I^{*}$ & $0.883(0.877-0.889)$ & $<0.00 I^{*}$ \\
\hline \multicolumn{9}{|l|}{ Community } \\
\hline Rural & Reference & & Reference & & Reference & & Reference & $<0.001 *$ \\
\hline Urban & $1.094(1.085-1.102)$ & $<0.001 *$ & $1.084(1.074-1.093)$ & $<0.001 *$ & $1.084(1.074-1.094)$ & $<0.00 I^{*}$ & $0.978(0.969-0.987)$ & $<0.001 *$ \\
\hline
\end{tabular}

Note: *p-value less of 0.05 was considered to be statistically significant.

Abbreviations: $\mathrm{OR}$, odds ratio; $\mathrm{Cl}$, confidence interval. 
In this study, we also analyzed antibiotic prescription rates according to age. The highest prevalence rates were observed in patients aged 71 years $(80.3 \%)$ followed by 4-year-old children (60.7\%). High prescription rates were also observed in other studies for the same age groups. ${ }^{5,27}$

In children, the high consumption of antibiotics could be due to their use to treat acute otitis media and upper respiratory tract infections, two of the most prevalent pediatric infections and a major source of inappropriate antibiotic prescribing in outpatient settings. ${ }^{28}$ In Europe, respiratory infections represent the second leading condition in children according to the Disability-Adjusted Life Years (DALY) score. ${ }^{6}$ However, this does not justify antibiotic overuse in this age group because common cold and sore throat are usually viral pathological conditions, self-limiting, and often easily self-managed. ${ }^{6}$ On the other hand, the implementation of clinical practice guidelines on the correct use of antibiotics to treat acute otitis media in children could avoid antibiotic therapy in $75 \%$ of the cases. ${ }^{6}$ In the older adults, higher antibiotic prescription rates are justified due to major health problems related to age. ${ }^{9}$

We also noted differences in antibiotic prevalence rates by sex, even though doctors are often impartial when prescribing drugs. The analysis showed a higher use of antibiotics in females when aged between 17 and 77 years. In an observational study conducted in Spain, antibiotic prescription rates were also higher in females aged between 15 and 65 years, and it was proportionately related to the frequency of medical visits. ${ }^{29,30}$ This relationship was later confirmed by one systematic review in $2016^{31}$ and a study conducted in the Italian region of Lombardy. ${ }^{5}$ Furthermore, other studies have also described higher antibiotic prescription rates in females, mainly due to the treatment of UTIs and a greater number of consultations. ${ }^{29}$ The prevalence rates trend was reversed in the older adults group. In males, the prevalence rate of antibiotic prescription was higher in the older adults, possibly because of a higher prevalence rate of chronic diseases, ${ }^{10}$ higher GPs consultation rates, and increased UTIs. ${ }^{31}$

Our analysis showed that the most prescribed antibiotic class were penicillins (ie, $57.8 \%$ of the population treated with antibiotics), followed by cephalosporins (30.5\%), macrolides $(27.9 \%)$, and fluoroquinolones $(26.9 \%)$. Indeed, penicillin is regarded as the first-choice drug when treating the most common infectious diseases in children ${ }^{6}$ and the most common respiratory drug infections in patients of all ages. ${ }^{5}$ Cephalosporins are used as a second-line therapy to treat the most common pediatric respiratory infections (ie, non-type I allergy to penicillin, treatment failure with antibacterial agents, and presence of severe symptoms). ${ }^{6}$ Of note, in some countries cephalosporins can only be used in hospitals. ${ }^{5}$ The overuse of oral cephalosporins is deemed to be unjustified since these are not recommended as a first-choice therapy for acute otitis media or pharyngotonsillitis, the two most common pediatric infectious diseases.

The most prescribed antibiotic class recorded in the three age groups was the Penicillin, except for patients aged $\geq 85$ years. According to a summary of European data on antibiotic consumption, penicillin was the most frequently used antibiotic throughout the countries, as we also saw in our study, while the use of other antibiotic classes varied considerably between countries. ${ }^{32}$ This trend has also been evidenced in other studies..$^{13,30,31,33}$

In the older adults $\geq 85$ years, the most prescribed antibiotic class were fluoroquinolones $(50.0 \%)$, thus confirming the results of a case control study conducted in Lombardy with the same population. ${ }^{5}$ The European Surveillance of Antimicrobial Consumption (ESAC) stated that the outpatient use of quinolones has been one of the fastest growing antibiotic classes since the start of their survey in 1997. In Italy, quinolones' highest prescription rate was recorded in 2009, and it continues to increase. ${ }^{26}$

Still, this does not justify fluoroquinolones' high prescription rates. Some evidence has demonstrated that their use for treating community-acquired respiratory tract infections, such as pneumonia, has increased in the United States, especially among patients aged $\geq 65$ years. ${ }^{5}$ However, in most European countries they are not recommended as first-line antibiotics for the treatment of lower respiratory tract infections in ambulatory care. The inappropriate use of fluoroquinolones, both in the older adults and to treat respiratory diseases, will inevitably lead to the emergence of resistant pneumococcus (Streptococcus pneumoniae) and resistant Gram-negative organisms. ${ }^{26}$

There are some limitations to our study. We performed a cross-sectional study using pharmaceutical records, which do not provide information about the diagnosis. Therefore, we were unable to explore the prescriptions' appropriateness because the diagnosis details were unavailable from our data sources.

The main strengths of our study lie in providing an overview of antibiotics use based on data sources with full coverage of the antibiotic prescriptions for a geographically defined, stable population. ${ }^{34,35}$ Antibiotic overuse and misuse contribute to the development of resistance, treatment failure, and high health costs. Local policies, following WHO's recommendations, should provide training and information to citizens and health care professionals to optimize health 
resources. Successful elements from other countries' activities should also be implemented. ${ }^{36,37}$

Modifying prescribing patterns is not an easy task. There is evidence that interventions combining educational material and prescribing feedback have successfully promoted appropriateness in drug prescription. ${ }^{38-40}$ Furthermore, synergies between different actors involved in healthcare delivery can help achieving better results.

\section{Conclusion}

Our study highlights antibiotic prescription differences at a municipality level in Campania and shows large differences within the same Local Health Unit (LHU) according to age. Despite national and international guidelines, aiming to optimize antibiotic prescription in community outpatients, antibiotic use in Campania is considerably higher than in other Italian regions or countries. ${ }^{2}$ Moreover, the use of second-line antibiotics is common. On the other hand, we evidenced that different levels of NHS expenditure exist within the same geographical area.

In such a context, this study could be a valid background to be used in planning formal audits concerning the prescribing approach to relevant clinical needs in community-acquired infections.

\section{Ethical Considerations}

All procedures performed in this study were in accordance with the current national law from the Italian Medicines Agency. The article does not contain clinical studies, and all patients' data were fully anonymized. For this type of study, formal consent was not required. Permission to use anonymized data for the present study was granted by the responsible authority, "Unità del Farmaco, Regione Campania".

\section{Acknowledgment}

This study is part of a set of initiatives to promote the appropriate drug use and drug safety in Campania Region.

\section{Author Contributions}

All authors contributed towards data analysis, drafting and critically revising the paper, gave final approval of the version to be published, and agreed to be accountable for all aspects of the work.

\section{Disclosure}

No funding was provided for this work and the authors report no conflicts of interest in this work.

\section{References}

1. Bruyndonckx R, Hens N, Aerts M, Goossens H, Molenberghs G, Coenen S. Measuring trends of outpatient antibiotic use in Europe: jointly modelling longitudinal data in defined daily doses and packages. J Antimicrob Chemother. 2014;69(7):1981-1986. doi:10.1093/jac/dku063

2. Adriaenssens N, Coenen S, Versporten A, et al. European Surveillance of Antimicrobial Consumption (ESAC): outpatient antibiotic use in Europe (1997-2009). J Antimicrob Chemother. 2011;66 (SUPPL. 6):3-12. doi:10.1093/jac/dkr190

3. Fleming-Dutra KE, Hersh AL, Shapiro DJ, et al. Prevalence of inappropriate antibiotic prescriptions among US ambulatory care visits, 2010-2011. JAMA. 2016;315(17):1864-1873. doi:10.1001/jama.2016.4151

4. Global Action Plan on Antimicrobial Resistance. Available from: http://www.wpro.who.int/entity/drug_resistance/resources/global_ action_plan_eng.pdf. Accessed 2018 May 7.

5. Franchi C, Sequi M, Bonati M, et al. Differences in outpatient antibiotic prescription in Italy's Lombardy region. Infection. 2011;39(4):299-308. doi:10.1007/s15010-011-0129-1

6. Rossignoli A, Clavenna A, Bonati M. Antibiotic prescription and prevalence rate in the outpatient paediatric population: analysis of surveys published during 2000-2005. Eur J Clin Pharmacol. 2007;63 (12):1099-1106. doi:10.1007/s00228-007-0376-3

7. Ong DSY, Kuyvenhoven MM, Van Dijk L, Verheij TJM. Antibiotics for respiratory, ear and urinary tract disorders and consistency among GPs. J Antimicrob Chemother. 2008;62(3):587-592. doi:10.1093/jac/dkn230

8. Klein EY, Makowsky M, Orlando M, Hatna E, Braykov NP, Laxminarayan R. Influence of provider and urgent care density across different socioeconomic strata on outpatient antibiotic prescribing in the USA. $J$ Antimicrob Chemother. 2014;70 (5):1580-1587. doi:10.1093/jac/dku563

9. Masiero G, Filippini M, Ferech M, Goossens H. Socioeconomic determinants of outpatient antibiotic use in Europe. Int $J$ Public Health. 2010;55(5):469-478. doi:10.1007/s00038-010-0167-y

10. Orzella L, Chini F, Giorgi Rossi P, Borgia P. Physician and patient characteristics associated with prescriptions and costs of drugs in the Lazio region of Italy. Health policy (New York). Elsevier Ireland Ltd. 2010;95(2-3):236-244.

11. Morgan DJ, Okeke IN, Laxminarayan R, Perencevich EN. Nonprescription antimicrobial use worldwide: a systematic review. Lancet Infect Dis. 2011;11(9):692-701.

12. Grigoryan L, Haaijer-Ruskamp FM, Burgerhof JGM, et al. Selfmedication with antimicrobial drugs in Europe. Emerg Infect Dis. 2006;12(3):452-459. doi:10.3201/eid1203.050992

13. Clavenna A, Sequi M, Bortolotti A, Merlino L, Fortino I, Bonati M. Determinants of the drug utilization profile in the paediatric population in Italy's Lombardy Region. Br J Clin Pharmacol. 2009;67 (5):565-571. doi:10.1111/bcp.2009.67.issue-5

14. Piovani D, Clavenna A, Cartabia M, Bonati M. Antibiotic and anti-asthmatic drug prescriptions in Italy: geographic patterns and socio-economic determinants at the district level. Eur $J$ Clin Pharmacol. 2014;70(3):331-337. doi:10.1007/s00228-013-1615-4

15. Rapporto OsMed [webpage on the Internet]. 2016. Available from: http://www.aifa.gov.it/content/luso-dei-farmaci-italia-rapporto-osmed -2016. Accessed June, 2018.

16. Russo V, Monetti VM, Guerriero F, et al. Prevalence of antibiotic prescription in southern Italian outpatients: real-world data analysis of socioeconomic and sociodemographic variables at a municipality level. Clinicoecon Outcomes Res. 2018;10:251-258. doi:10.2147/CEOR. S161299

17. Legge 16.11.2001, n. 405. "Conversione in legge, con modificazioni, del decreto-legge 18 settembre 2001, n. 347, recante interventi urgenti in materia di spesa sanitaria". Gazzetta Ufficiale 17. novembre 2001, n. 268. 
18. Demo-Geodemo. Mappe, Popolazione, Statistiche Demografiche dell'ISTAT. Available from: http://demo.istat.it/. Accessed 2018, May 7.

19. WHOCC-ATC/DDD Index. Available from: https://www.whocc.no/ atc_ddd_index/. Accessed 2018, May 7.

20. Iolascon G, Gimigliano F, Moretti A, et al. Rates and reasons for lack of persistence with anti-osteoporotic drugs: analysis of the Campania region database. Clin Cases Miner Bone Metab. 2016;13(2):126-129. doi:10.11138/ccmbm/2016.13.2.127

21. Cammarota S, Bruzzese D, Catapano AL, et al. Lower incidence of macrovascular complications in patients on insulin glargine versus those on basal human insulins: a population-based cohort study in Italy. Nutr Metab Cardiovasc Dis. 2014;24(1):10-17. doi:10.1016/j. numecd.2013.04.002

22. Scala D, Menditto E, Caruso G, et al. Are you more concerned about or relieved by medicines? An explorative randomized study of the impact of telephone counseling by pharmacists on patients' beliefs regarding medicines and blood pressure control. Patient Educ Couns. 2018;101(4):679-686. doi:10.1016/j.pec.2017.12.006

23. Putignano D, Bruzzese D, Orlando V, Fiorentino D, Tettamanti A, Menditto E. Differences in drug use between men and women: an Italian cross sectional study. BMC Womens Health. 2017;17(1):73. doi:10.1186/s12905-017-0424-9

24. Guerriero F, Orlando V, Monetti VM, Russo V, Menditto E. Biological therapy utilization, switching, and cost among patients with psoriasis: retrospective analysis of administrative databases in Southern Italy. Clinicoecon Outcomes Res. 2017;1(9):741-748. doi:10.2147/CEOR.S147558

25. Scala D, Menditto E, Armellino MF, et al. Italian translation and cultural adaptation of the communication assessment tool in an outpatient surgical clinic. BMC Health Serv Res. 2016;16(1):163. doi:10.1186/s12913-016-1411-9

26. Adriaenssens N, Coenen S, Versporten A, et al. European Surveillance of Antimicrobial Consumption (ESAC): outpatient quinolone use in Europe (1997-2009). J Antimicrob Chemother. 2011;66 (SUPPL. 6):47-56. doi:10.1093/jac/dkr457

27. Augustin J, Mangiapane S, Kern WV. A regional analysis of outpatient antibiotic prescribing in Germany in 2010. Eur J Public Health. 2015;25(3):397-399. doi:10.1093/eurpub/ckv050

28. Nitzan O, Low M, Lavi I, Hammerman A, Klang S, Raz R. Variability in outpatient antimicrobial consumption in Israel. Infection. 2010;38(1):12-18. doi:10.1007/s15010-009-9065-8

29. Serna MC, Real J, Ribes E, Marsal JR, Godoy P, Galván L. Determinantes de la prescripción de antibióticos en atención primaria. Enferm Infecc Microbiol Clin. 2011;29(3):193-200. doi:10.1016/j.eimc.2010.09.012
30. Malo-Fumanal S, Rabanaque-Hernández MJ, Feja-Solana C, LallanaAlvarez MJ, Armesto-Gómez J, Bjerrum L. Differences in outpatient antibiotic use between a Spanish region and a Nordic country. Enferm Infecc Microbiol Clin Elsevier Doyma. 2014;32(7):412-417. doi:10.1016/j.eimc.2013.10.002

31. Schröder W, Sommer H, Gladstone BP, et al. Gender differences in antibiotic prescribing in the community: a systematic review and meta-analysis. J Antimicrob Chemother. 2016;71(7):1800-1806. doi: $10.1093 / \mathrm{jac} / \mathrm{dkw} 054$

32. Euro-Cdc. Summary of the latest data on antibiotic consumption in the European Union Antibiotic consumption in Europe. Eoro-Cdc. 2012;19(November): 1-8.

33. Di Martino M, Lallo A, Kirchmayer U, Davoli M, Fusco D. Prevalence of antibiotic prescription in pediatric outpatients in Italy: the role of local health districts and primary care physicians in determining variation. A multilevel design for healthcare decision support. BMC Public Health. 2017;17(1):1-8. doi:10.1186/s12889017-4905-4

34. Iolascon G, Gimigliano F, Orlando V, et al. Osteoporosis drugs in real-world clinical practice: an analysis of persistence. Aging Clin Exp Res. 2013;25 Suppl 1:S137-S141. doi:10.1007/s40520-0130127-5.

35. Menditto E, Cahir C, Aza-Pascual-Salcedo M, et al. Adherence to chronic medication in older populations: application of a common protocol among three European cohorts. Patient Prefer Adherence. 2018;12:1975. doi:10.2147/PPA.S164819

36. Illario M, Vollenbroek-Hutten M, Molloy DW, et al. Active and healthy ageing and independent living. $J$ Aging Res. 2015;2015:542183. doi:10.1155/2015/542183

37. Illario M, Vollenbroek-Hutten MMR, Molloy DW, Menditto E, Iaccarino G, Eklund P. Active and healthy ageing and independent living 2016. J Aging Res. 2016;2016:8062079. doi:10.1155/2016/ 8062079

38. Hux JE, Melady MP, DeBoer D. Confidential prescriber feedback and education to improve antibiotic use in primary care: a controlled trial. CMAJ. 1999;161(4):388-392.

39. Coretti S, Romano F, Orlando V, et al. Economic evaluation of screening programs for hepatitis $\mathrm{C}$ virus infection: evidence from literature. Risk Manag Healthc Policy. 2015;8:45-54. doi:10.2147/ RMHP.S56911.

40. Menditto E, Orlando V, Coretti S, Putignano D, Fiorentino D, Ruggeri M. Doctors commitment and long-term effectiveness for cost containment policies: lesson learned from biosimilar drugs. Clinicoecon Outcomes Res. 2015;7:575-581. doi:10.2147/CEOR. $\mathrm{S} 88531$
Risk Management and Healthcare Policy

\section{Publish your work in this journal}

Risk Management and Healthcare Policy is an international, peerreviewed, open access journal focusing on all aspects of public health, policy, and preventative measures to promote good health and improve morbidity and mortality in the population. The journal welcomes submitted papers covering original research, basic science, clinical \& epidemiological studies, reviews and evaluations, guidelines, expert opinion and commentary, case reports and extended reports. The manuscript management system is completely online and includes a very quick and fair peer-review system, which is all easy to use. Visit http://www.dovepress.com/testimonials.php to read real quotes from published authors. 